\title{
Particle deposition from aerosol flow inside a T-shaped micro-mixer
}

\author{
Michael Heim, Robert Wengeler, Hermann Nirschl and \\ Gerhard Kasper \\ Institut für Mechanische Verfahrenstechnik und Mechanik, Universität Karlsruhe (TH), \\ D-76128 Karlsruhe, Germany \\ E-mail: robert.wengeler@mvm.uka.de and michael.heim@mvm.uka.de
}

Received 23 August 2005, in final form 17 November 2005

Published 7 December 2005

Online at stacks.iop.org/JMM/16/70

\begin{abstract}
A T-shaped micro-reactor was used to mix a particle laden gas stream (an 'aerosol') and a clean gas stream, here with the objective of determining particle losses to the inside walls of the reactor (and hence the potential for clogging) as a function of operating conditions. Losses were determined with established on-line concentration measurement techniques, using monodisperse sodium chloride particles and vitamin-E-acetate droplets of variable size range between 10 and $700 \mathrm{~nm}$. Measured losses range between 10 and $95 \%$, depending on flow rate and particle size, and can be modeled with the particle Stokes number. CFD and particle trajectory modeling confirm that particle impact on the walls of the inlet and the mixing zones is the predominant particle deposition mechanism, induced by the local, sharp curvature of streamlines. The modeling points to ways of optimizing the inlet geometry of the mixer to reduce impaction losses significantly.
\end{abstract}

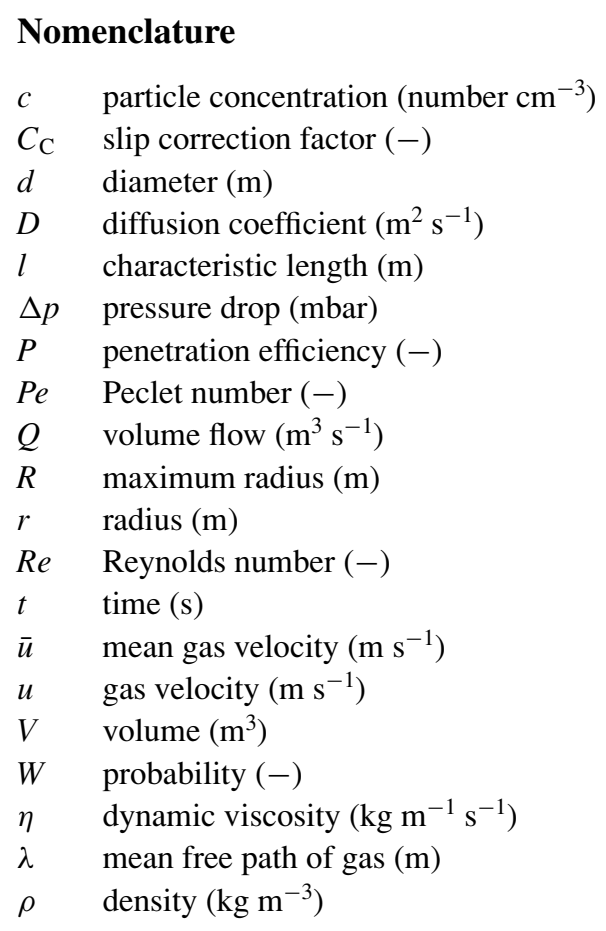

\begin{tabular}{ll}
\multicolumn{2}{l}{ Subscripts } \\
0 & initial value \\
1 & mixer inlet 1 \\
2 & mixer inlet 2 \\
3 & mixer outlet \\
$i$ & timestep index \\
$\mathrm{P}$ & particle \\
$\mathrm{r}$ & residence \\
mix & due to mixing
\end{tabular}

\section{Introduction}

Designing particle-based materials with complex product properties is often better achieved through continuous, multistage processes, rather than with classical unit operations [1].

Such modern chemical engineering techniques frequently involve aerosol flows, which have to be integrated into the overall process. Micro-reactor technology has already demonstrated its suitability for many liquid phase applications and shows considerable potential to improve certain tasks in aerosol processing, e.g., by promoting rapid mixing of aerosol streams to produce more uniform dispersions and coatings. 


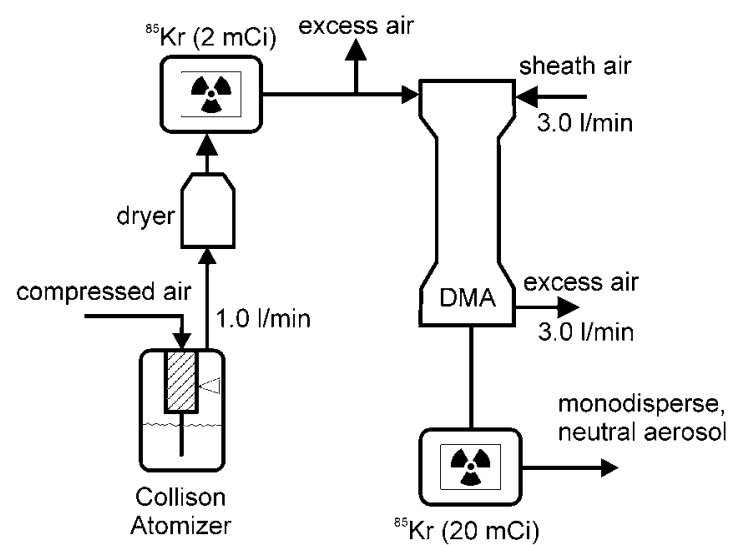

Figure 1. Experimental setup to produce monodisperse sodium chloride aerosol particles.

Combining such process steps with micro-technology is especially interesting for innovative products requiring multifunctional materials based on complex particles or particulate structures such as pharmaceutical agents [2, 3], scents or functionalized active agents for cosmetics, because the required amounts are small, even considering international markets. However, the possibilities and potential limitations of micro-technology-based aerosol processes have not been studied systematically. One potential drawback for industrial applications of aerosol processing in micro-reactors is the obvious need to minimize internal particle deposition.

The large surface-to-volume ratio which is advantageous in many applications demands special attention on unwanted particle deposition on the micro channel walls. As a first step to understand aerosol flow inside a micro-device, an experimental and theoretical study of particle deposition in a simple static T-shaped micro-mixer has been conducted, to derive constraints and design parameters for micro-reactors capable of handling aerosols.

\section{Experimental setup}

\subsection{Aerosol generation}

Monodisperse sodium chloride or vitamin-E-acetate aerosols were used for the experimental determination of the particle losses in the micro-mixer. The salt particles between 10 and $900 \mathrm{~nm}$ were generated by atomizing $\mathrm{NaCl}$ solutions of different concentration with a collison nebulizer [5] and drying the droplets either in a silica gel diffusion dryer or in a Permapure ${ }^{\circledR}$ tube-dryer (figure 1). The somewhat larger vitamin-E (tocopherol acetate) droplets in the size range from 300 to $900 \mathrm{~nm}$ were produced with the same nebulizer, but without subsequent drying. The aerosol was then classified with a differential mobility analyzer (DMA; Model Grimm 5.4-900 [6]) to obtain singly charged aerosol particles with a narrow size distribution. To further increase the monodispersity, the small fraction of larger particles with multiple charges always present in DMA classified aerosols [7] was removed with an impactor (not shown in figure 1) positioned upstream of the DMA. Finally, to avoid the influence of electrostatic deposition effects within the mixer, this aerosol was neutralized electrically through a second

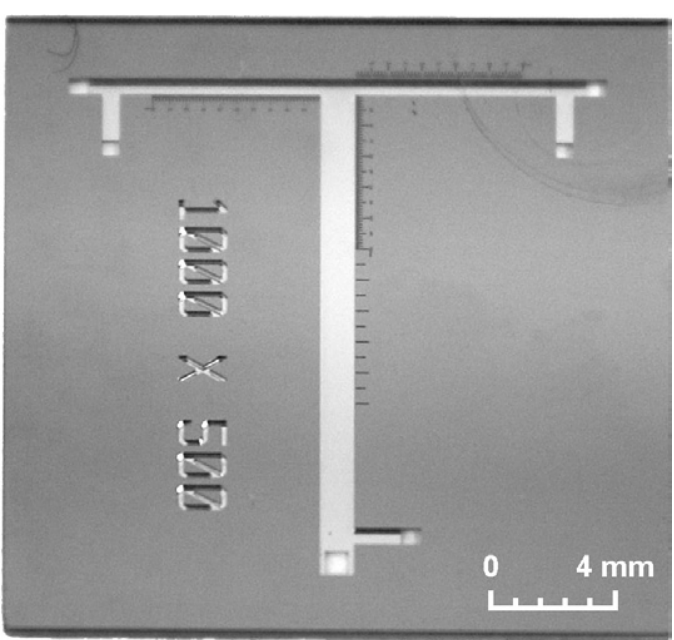

Figure 2. Photograph of the silicon-based T-shaped micro-mixer with a channel depth of $300 \mu \mathrm{m}$ and channel widths in the inlet and mixing channels of 500 and $1000 \mu \mathrm{m}$, respectively.

Krypton- 85 charger, resulting in a defined aerosol charge distribution [8] with mostly uncharged particles.

It should also be pointed out that it is customary in aerosol technology to express particle sizes in terms of the equivalent diameter of the device with which they were measured, in this case the DMA. Hence, the particle size in subsequent graphs is given in terms of mobility equivalent diameter. For spherical droplets and $\mathrm{NaCl}$ particles of near-spherical shape, the mobility equivalent diameter is practically identical with the physical diameter of the particle.

The static T-shaped micro-mixer (courtesy of the Institute of Microsystems Technology, University of Freiburg) is fabricated using standard silicon technologies, with RIEetched rectangular channels of $1000 \times 500 \times 300 \mu \mathrm{m}$ (width of mixing channel $\times$ width of inlet channels $\times$ height of all channels, respectively); the fluidic connections from the rear are etched with $\mathrm{KOH}$. The mixer is covered by a Pyrex glass wafer and is mounted on a support to allow the necessary fluidic connections to be attached [9]. Figure 2 shows a picture of the micro-mixer used.

Particle losses in the micro-mixer were measured with the setup shown in figure 3. The monodisperse aerosol flow is split; one part is fed directly into one branch of the T-mixer, whereas the other is passed through a high-efficiency particle filter before entering through the opposite branch of the mixer. Both flows then recombine and are drawn through the device with a constant flow rate of $0.31 \mathrm{~min}^{-1}$, using the internal pump of the attached condensation particle counter (CPC; Model Grimm 5.403 [6]), which is capable of counting particles down to a size of $7.5 \mathrm{~nm}$. The particle number concentration used was always below $10^{4} \mathrm{~cm}^{-3}$, so that only the more accurate single particle count mode of the CPC was used [6]. Tubes of equal length were used for the concentration measurements before and after the mixer, to minimize errors resulting from any particle losses inside the ducts.

The actual mixing ratio was determined by measuring the flow rate of the particle-free air stream with a sensitive flow meter; it could be varied by raising the pressure drop in the particle-free line with a needle valve. Furthermore, the total 


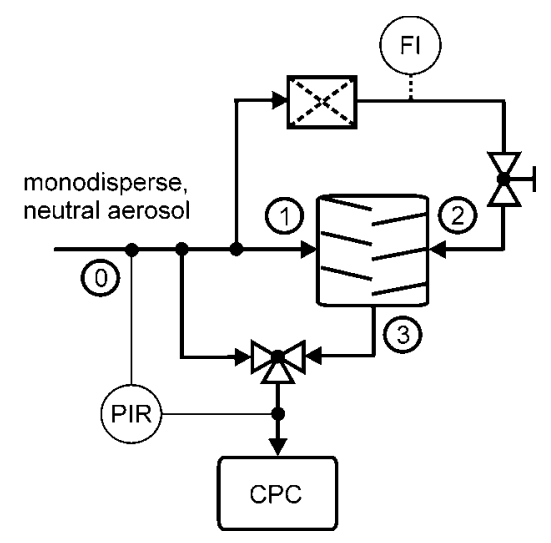

Figure 3. Experimental setup used for the measurement of the particle losses within the micro-mixer.

pressure drop across the mixer was measured, because it gives an indication of the clogging behavior.

\section{Experiments performed}

With the experimental setup described above, the penetration efficiency of the mixer was measured for a mixing ratio of 1.35:1, using monodisperse fractions of $\mathrm{NaCl}$ particles in the size range between 10 and $900 \mathrm{~nm}$ and with vitamin-E droplets between 300 and $900 \mathrm{~nm}$. For each particle size, the concentration before and after the mixer, the pressure drop and the flow rate of the particle-free air were measured five times and the mean value of the penetration efficiency $P$ was calculated, according to

$$
P=\frac{c_{3}}{c_{0}} \frac{Q_{1}+Q_{2}}{Q_{1}}
$$

where $c_{3} / c_{0}$ denotes the ratio of the mixer outlet concentration to the bypass line concentration; $Q_{1}$ and $Q_{2}$ are the volume flow rates of the two flows entering the mixer. To show the reproducibility of the data, the measurements were repeated twice, two days apart. Particle losses are obtained by calculating $1-P$.

The increase in pressure drop and penetration efficiency caused by clogging was also measured, using DMA-classified $\mathrm{NaCl}$ particles with an electrical mobility diameter of $200 \mathrm{~nm}$ and a number concentration of approximately $10^{5} \mathrm{~cm}^{-3}$. For these experiments, the mixing ratio was allowed to change during a run, driven by the rising pressure drop in the particle laden branch of the mixer. However, the mixing ratio was measured at regular intervals and taken into account for the calculation of the penetration efficiency according to equation (1).

\section{Results and discussion}

The penetration efficiency measurements shown in figure 4 reveal a relatively broad maximum, with increasing particle losses in the size range below about $50 \mathrm{~nm}$ and above about $200 \mathrm{~nm}$ (for the droplets about $400 \mathrm{~nm}$ ). A slight but systematic difference between the two types of particles will be explained below, in terms of density.

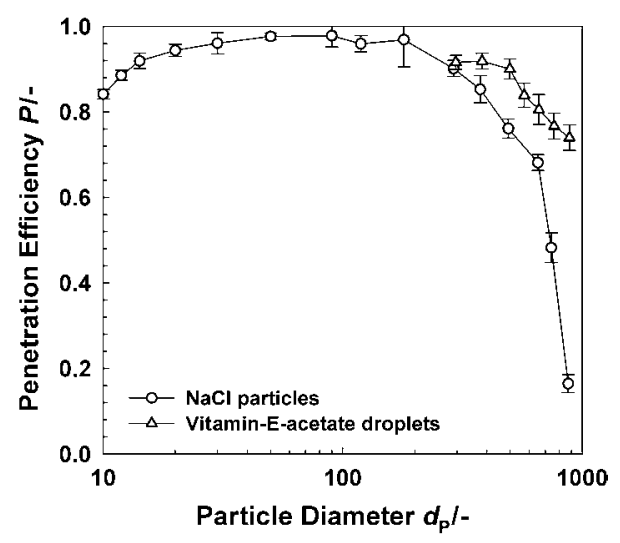

Figure 4. Penetration efficiency of the micro-mixer measured with monodisperse $\mathrm{NaCl}$ particles and vitamin-E droplets of varying size for a total flow rate of $0.31 \mathrm{~min}^{-1}$ and a mixing ratio of 1.35:1.

The losses of very small particles are mostly caused by particle diffusion and usually modeled in terms of a dimensionless parameter called Peclet number $(\mathrm{Pe})$,

$$
P e=\frac{u l}{D_{\mathrm{P}}},
$$

where $u$ denotes the mean gas velocity (and hence the residence time of the particles in the reactor), $l$ is a characteristic geometric dimension (e.g. the hydraulic diameter of the mixer channel) and $D_{\mathrm{P}}$ is the particle diffusion coefficient. With increasing $P e$ number, the diffusional process becomes less important and can apparently be neglected in the current case above $50 \mathrm{~nm}$, where $P e$ exceeds $5 \times 10^{6}$. Diffusion losses are quite small, given the short residence time of particles in the reactor.

The losses of larger particles are most likely induced by inertial particle deposition in regions of the mixer or the fluid inlet where the channel has sharp angles. In this size region, particles cannot follow the streamlines due to their inertia, and impact on the surface of the wall where they adhere. Rebound of such small particles does not occur until much higher impact velocities, and their sticking probability can be assumed to be unity, because van-der-Waals adhesion force exceeds the fluid drag in the boundary sub-layer.

The difference in penetration efficiency between the two materials observed in figure 4 is most likely caused by their different densities $\left(2.16 \mathrm{~g} \mathrm{~cm}^{-3}\right.$ for sodium chloride versus $0.96 \mathrm{~g} \mathrm{~cm}^{-3}$ for the vitamin-E). Particle deposition in the inertia-dominated regime is modeled in terms of the Stokes number (St) (essentially a dimensionless particle size), which could be regarded as the particles' stop distance in relation to an obstacle geometry,

$$
S t=\frac{\rho_{\mathrm{P}} C_{\mathrm{C}}\left(d_{\mathrm{P}}\right) d_{\mathrm{P}}^{2} u}{18 \eta d_{\mathrm{o}}},
$$

where $C_{\mathrm{C}}\left(d_{\mathrm{P}}\right)$ is the slip correction factor given by

$$
C_{\mathrm{C}}\left(d_{\mathrm{P}}\right)=1+\frac{2 \lambda}{d_{\mathrm{P}}}\left[1.257+0.4 \exp \left(\frac{-0.55 d_{\mathrm{P}}}{\lambda}\right)\right],
$$

which is in turn a function of particle size and mean free path of the carrier gas $\lambda[10] ; \rho_{\mathrm{P}}$ denotes the bulk density of the particle material, $u$ the gas velocity, $\eta$ the dynamic viscosity of the surrounding gas and $d_{\mathrm{o}}$ the characteristic dimension of 


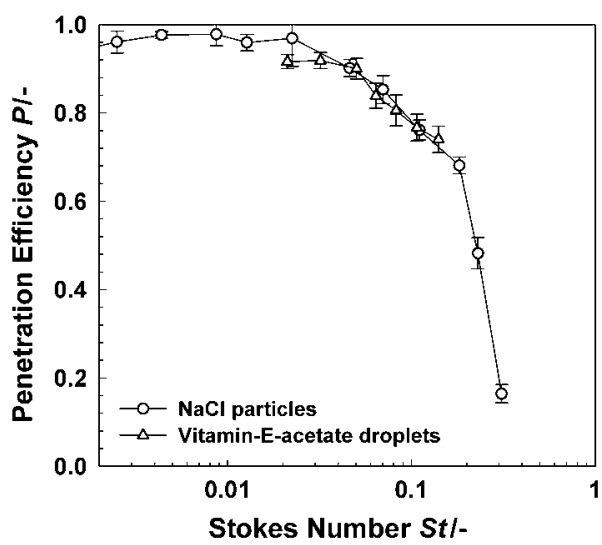

Figure 5. Penetration efficiency of the micro-mixer versus Stokes number, for monodisperse $\mathrm{NaCl}$ particles and vitamin-E droplets of varying size (total flow rate of $0.31 \mathrm{~min}^{-1}$, mixing ratio of 1.35:1).

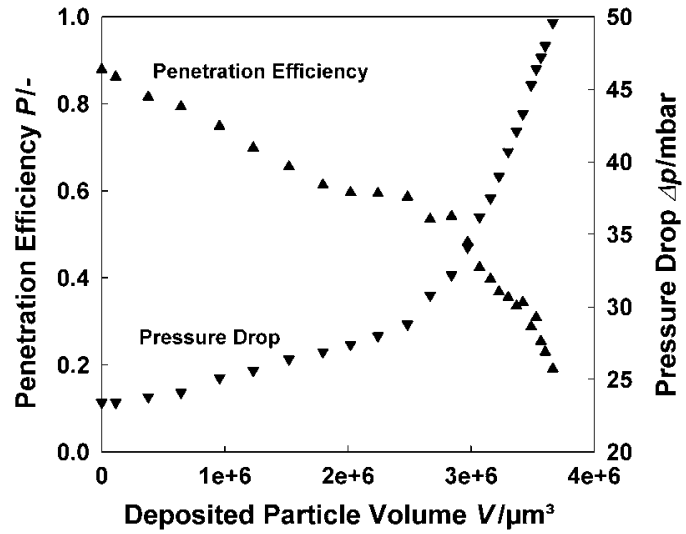

Figure 6. Variation in pressure drop and penetration efficiency of the micro-mixer caused by progressive clogging with (DMA classified) $200 \mathrm{~nm} \mathrm{NaCl}$ particles, as a function of the calculated deposited particle volume.

the obstacle, in this case the hydraulic diameter of the mixer inlet. By replotting the data of figure 4 in terms of $S t$ number, one can more accurately compare the penetration efficiencies for both particle materials examined. Figure 5 shows clearly that both materials now closely follow the same functional behavior, indicating that the losses are principally driven by inertial particle deposition. Furthermore, the figure permits conversions to other particle sizes, densities, flow rates and channel dimensions.

According to figure 5, a useful working range of the mixer is limited to a range of Stokes and Peclet numbers where losses are minimal, in our case between approximately $50 \mathrm{~nm}$ at the low end $\left(\mathrm{Pe}>5 \times 10^{6}\right)$, and a few hundreds of nanometer at the upper end $(S t<0.02)$.

A major problem related with particle deposition in a micro-mixer is clogging, since it will change the mixer's flow characteristics and can lead to a poor performance. The effect of increased particle deposition can be observed clearly in figure 6 , where the change in the penetration efficiency and the pressure drop across the mixer is shown as a function of the deposited particle volume. With increasing deposited particle volume, the penetration efficiency decreases whereas the pressure drop increases. The deterioration of the penetration efficiency is caused by the growing particle

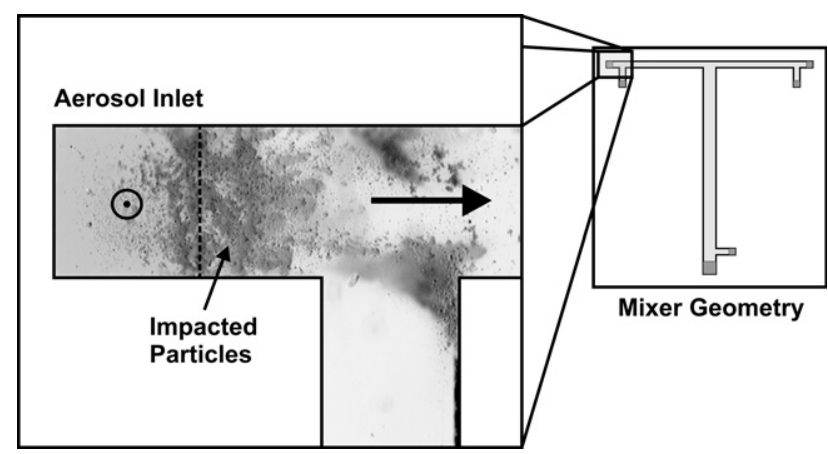

Figure 7. Photograph (negative) of deposition structure formed by $200 \mathrm{~nm} \mathrm{NaCl}$ particles in the inlet region of the micro-mixer. Inlet (on the left of the image) is vertical, main flow is in the plane of the image.

structures formed by impacted particles, which results in even stronger redirections of the flow and hence to an increased deposition of particles. The deposited particle volume between two measurements is calculated according to equation (5) from the number concentration of the particles entering the mixer $c_{0}$, the volume flow $Q_{1}$, the time between two measurements $(\Delta t)$ and the average penetration efficiency $(P)$ between two measurements:

$$
\Delta V_{i}=c_{0} Q_{1} \Delta t P \frac{\pi}{6} d_{\mathrm{P}}^{3},
$$

where $c_{0}, P$ and $Q_{1}$ are calculated as the mean value of the respective parameters for each time interval $\Delta t(i)$ :

$$
c_{0}=\frac{c_{0, i}+c_{0, i-1}}{2} \quad P=\frac{P_{i}+P_{i-1}}{2} \quad Q_{1}=\frac{Q_{1, i}+Q_{1, i-1}}{2} .
$$

Figure 7 gives a visual impression of the particle deposit at the inlet of the micro-mixer, where the flow makes a $90^{\circ}$ bend to enter the rectangular $500 \mu \mathrm{m} \times 300 \mu \mathrm{m}$ channel. As expected, the largest deposit is found immediately after the mixer entrance, on the Pyrex glass plate that forms the upper wall of the mixer. To prevent these particle losses, a redesign of the fluid inlet is necessary to avoid such bends and to allow a smooth, co-axial entrance into the mixer.

Unfortunately, losses occur also in the mixing region, as seen in figure 8 , most likely caused by the formation of vortices in the mixing zone [4], which prevents inertial particles from following the streamlines of these vortices and impacts them on the surrounding walls. The exit of the mixed flow region also shows visual signs of some particle deposition, which is, however, minor compared to the deposits found at the inlet and in the mixing zone.

\section{Fluid dynamics simulation of the micro-mixer}

A numerical calculation simulating the laminar mixing flow in the T-shaped static mixer was performed using the commercial CFD-code FLUENT 6.2. Using laminar flow the results of former studies on liquid flow $[4,11,12]$ can be confirmed to hold true for gas phase flows. The simulations are based on a three-dimensional T-shaped micro channel geometry for the numerical grid. Laminar fluid dynamics calculations with Dirichlet boundary conditions at the flow inlet and outlet are 


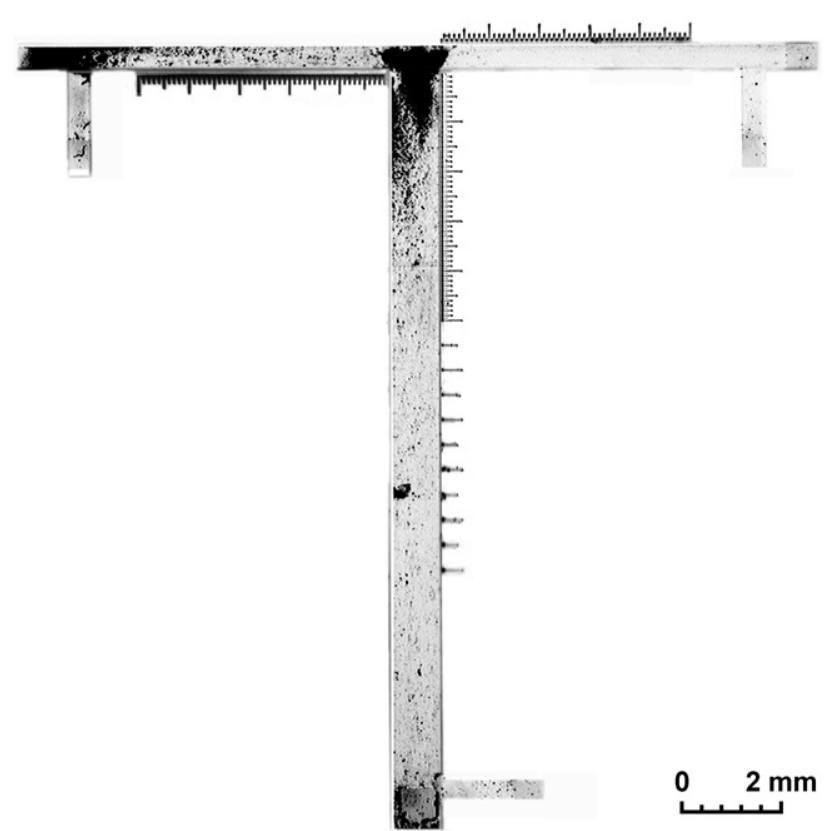

Figure 8. Photograph (negative) of the deposition structure (dark colors represent deposited particles) on the walls of the micro-mixer $(200 \mathrm{~nm} \mathrm{NaCl}$ particles, loading time of $240 \mathrm{~min}$ with an initial particle concentration of about $10^{5} \mathrm{~cm}^{-3}$, consistent with a deposited particle volume of $3.7 \times 10^{6} \mu \mathrm{m}^{3}$ ).

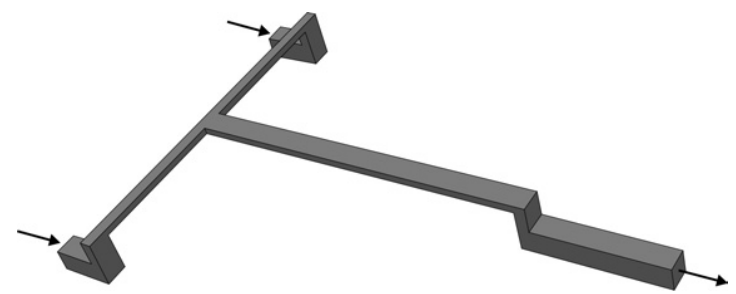

Figure 9. 3D image of the mesh geometry generated for CFD simulations with arrows indicating flow inlet and outlet boundaries.

performed to visualize stream lines. For different ranges of Reynolds numbers [11], the stream lines show stratified laminar flow $\left(R e_{\mathrm{M}}<10\right)$, vortex flow $\left(10<R e_{\mathrm{M}}<80\right)$ and stationary engulfment flow $\left(80<R e_{\mathrm{M}}<250\right)$. For higher Reynolds numbers, non-stationary laminar flow leads to engulfment flow regimes within the mixing channel.

These preliminary numerical calculations do not explain the deposition of particles at the inlet, where most of the deposition occurred. Therefore more comprehensive numerical studies have included the inlets and outlet of the fluidic support into the mesh of the T-shaped mixer model, with channel dimensions of $1000 \times 500 \times 300$ and a total width and length of the micro channels of $16 \mathrm{~mm}$ each. The inlets and outlet are $1 \mathrm{~mm}$ rectangular channels which enter the mixer plane at a $90^{\circ}$ angle and another $90^{\circ}$ angle toward the fluid support as shown in figure 9.

The spatial discretization amounts to 1.1 million nodes with 1 million hexahedral cells, which possess a higher node density in areas with higher velocity gradients. This was achieved by an adaptation of the mesh in accordance with the increasing velocity gradient. To enable a more detailed study of the flow field in the inlet and mixing zones, the grid resolution in these particular zones was especially emphasized.
A good agreement was found for the integral pressure drop in the simulated geometry of $\Delta p=25.6 \mathrm{mbar}$, which corresponds to the experimental value ( $\Delta p=24 \mathrm{mbar}$ ) of the particle-free micro-mixer. The pressure loss due to mixing accounts for $\Delta p_{\text {mix }}=5.1$ mbar of the total pressure loss calculated with the method presented by Engler [11]. The Reynolds number in the mixing micro channel equals $R e=444$ where laminar vortices are expected from former studies [11, 12].

When the inlet geometry of the micro-mixer and the fluidic connections and the resultant increase in velocity of the flow entering the micro channels are taken into account, it can be seen that vortices are induced in the two inlet branches of the Tshape. These vortices are not completely stratified to laminar flow before entering the mixing zone, as shown in figure 10 by iso-velocity lines at different positions in the inlet channel.

The high curvature of stream lines of the vortices induces particle deposition in this region due to inertial effects. The (experimentally confirmed) high deposition rate of particles at the beginning of the micro channel affirms the existence of such vortices from the inlet. Furthermore a decreasing amount of deposited particles from the inlet to the mixing zone can be observed in figure 8 , which could be a result of the ongoing laminarization of the flow.

\section{Particle track simulations}

For comparison with the experimental data, the particle penetration probability through the mixer and its fluidic connections was calculated using the discrete phase model (DPM) of FLUENT to simulate particle trajectories. The penetration probability was derived from the ratio of the number of particles passing through the mixer to that entering the system. Particle diffusion was neglected, and only losses due to inertial effects were implemented in the equation of motion.

FLUENT provides the option of controlling the starting point of each particle trajectory by a user-defined function (UDF). Thus, it is possible to displace the initial position according to a given equation representing the desired density distribution of particles in the channel. In our case, we assumed an equal particle concentration across the inlet area, given a particle density distribution that can be derived directly from the prevailing flow field. The flow field is assumed to follow the law of Hagen-Poiseuille. The density distribution used is represented by equation (7), where $r$ denotes the starting radius of a given particle track, $R$ the enclosed radius of the channel given by the inlet geometry and $W$ the corresponding probability of a particle being injected on the radius $r$ :

$$
W(r)=1-\left(\frac{r}{R}\right)^{2} \quad r \leqslant R .
$$

Before considering the particle density distribution, the injection coordinates for the actual particle track must be determined. Since all particle tracks start at a given plane perpendicular to the flow direction, only two coordinates are required. These are generated using a random number generator (Mersenne twister, [13]), and the generated coordinates are verified to represent a point within the specified circular entrance area by checking the radial distance to the center. 


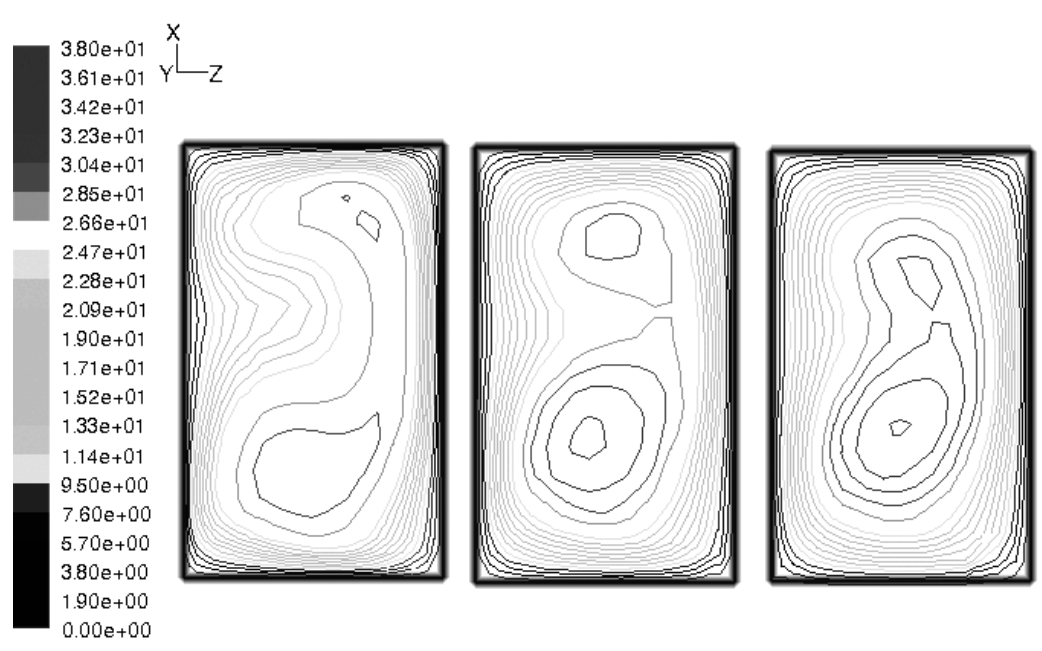

Figure 10. Iso-velocity lines in the inlet micro channel of the T-shaped mixer at positions 2,4 and $6 \mathrm{~mm}$ from the inlet $(6,4$ and $2 \mathrm{~mm}$ from the mixing axis), incompletely stratifying from left to right to a laminar flow profile.

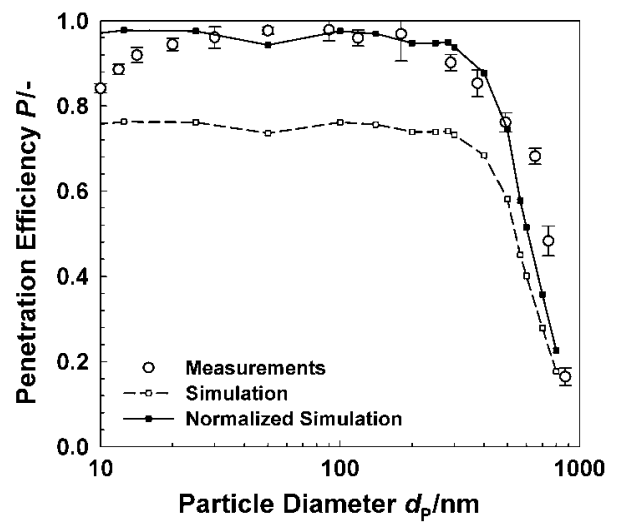

Figure 11. Comparison of the measured and simulated penetration efficiencies of the micro-mixer. The dots on the simulation curves represent the particle sizes, for which particle track calculations were performed.

To achieve the desired uniform particle concentration across the inlet area, the probability for every starting point has to be determined and starting points with lower probabilities are discarded. For every generated starting point the value according to equation (6) was calculated and then compared to another newly generated random number $(0-1)$. If the random number is larger than the value given by the equation, the generated position is declared invalid and discarded.

The penetration efficiency derived from the simulations (the dashed curve) is shown in figure 11, together with the previously shown experimental data for $\mathrm{NaCl}$ particles. The simulated penetration curve apparently never reaches $100 \%$, even for very small, completely inertialess particles. This is due to the insufficient resolution of the grid in the outlet region of the mixer, which lowers the penetration efficiency for particles in vortices that are not completely resolved by the numerical grid. Since it was not possible to increase the resolution without resorting to a mainframe computer, the simulated results were normalized with regard to the value obtained at the plateau-this is the solid curve shown in figure 11. After normalization the agreement with the

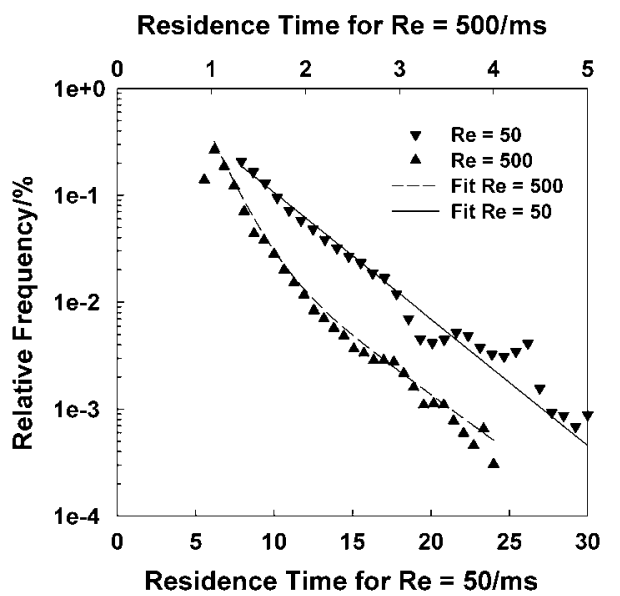

Figure 12. Computed particle residence time distribution for two different Reynolds numbers.

experimental data is quite good, with regard to the effect of particle deposition by impaction. At the lower end of the particle size range, particle deposition by diffusional effects is of course neglected.

Another important factor for particle processing in a micro-reactor is the residence time distribution (RTD) within the micro-structure. Figure 12 shows the very short residence times of $t_{\mathrm{r}}=1.4 \mathrm{~ms}$ and $11.1 \mathrm{~ms}$, for $R e=500$ and $R e=50$ respectively, obtained from the numerical simulations. For small Reynolds numbers, e.g. $R e=50$, no vortices occur in the flow and the residence time follows an exponential form. A higher Reynolds number, e.g. $R e=500$ typical also for the experimental study, leads to vortex structures with backflow areas which act as a hold up for the particles. This results in a residence time distribution which can be expressed as the sum of two exponential functions. The deviation from the fit lines which occur at high residence times is believed to be an artifact of the velocity gradients at the near-wall region. Due to the excessive computational requirements these could not sufficiently be resolved by the numerical grid. A statistical origin of the observed phenomena is unlikely since an increase 
in the total particle number used for the simulation does not show any influence on the functional behavior.

\section{Conclusions}

Operational characteristics and constraints for a microreactor handling submicron and nanoscale aerosols have been determined, both from measurements and simulations of particle losses and residence times.

It was shown that particle deposition above about $200 \mathrm{~nm}$ is significant and driven by inertial impaction, mostly induced by a sudden change in flow direction at the inlet, and by vortices in the mixing zone. The numerical study shows that laminar vortices occur even at low Reynolds numbers and lead to a higher particle deposition on the walls in the mixing zone.

While the inlet zone can be redesigned, the vortices cannot be avoided because vortex or engulfment flow in the mixing zone is needed to reduce mixing time. Therefore particle deposition in that region has to be taken into account in process design, and cleaning routines may be required to regenerate the micro-reactor.

This work shows that micro-reactors are suitable for aerosol processes, if suitably designed and operated. It is hoped that this work will encourage the use of micro-reactors for aerosol processing.

\section{Acknowledgments}

We gratefully acknowledge financial support from the State of Baden-Württemberg under the priority program 'Microtechnology Supported Integrated Processes'. We are grateful to the group of Professor Woias from the University of Freiburg, Germany, and specifically Dr Kockmann and Mr Engler for supplying the micro-mixers and the fluidic support.

\section{References}

[1] Strohrmann M 2004 The vision of an integrated process Proc. Symp. Process Technology beyond Unit Operations (Karlsruhe)

[2] Fukumori Y, Leuenberger H and Horio M 2004 Special issue on pharmaceutical particle formation Preface Powder Technol. 141171

[3] Lähde A, Watanabe W, Kauppinen E I and Brown D P 2004 Aerosol synthesis of inhalation powders Proc. Int. Congr. for Particle Technology (Nürnberg)

[4] Kockmann N, Föll C and Woias P 2003 Flow regimes and mass transfer characteristics in static micro-mixers Proc. SPIE 4982 319-29

[5] May K R 1973 The collison nebulizer: description, performance and application J. Aerosol Sci. 4 235-43

[6] Heim M, Kasper G, Reischl G P and Gerhart C 2004 Performance of a new commercial electrical mobility spectrometer Aerosol Sci. Technol. 38 3-13

[7] Knutson E O and Whitby K T 1974 Aerosol classification by electric mobility: apparatus, theory, and applications J. Aerosol Sci. 6 443-51

[8] Fuchs N A 1963 On the stationary charge distribution on aerosol particles in a bipolar ionic atmosphere Pure Appl. Geophys. 56 185-93

[9] Kockmann N, Engler M, Föll C and Woias P 2003 Liquid mixing in static micro mixers with various cross sections Proc. 1st. Int. Conf. on Micro- and Minichannels (Rochester NY, USA)

[10] Davies C 1945 Definitive equation for the fluid resistance of spheres Proc. R. Soc. 57259

[11] Engler M, Kiefer T, Kockmann N and Woias P 2004 Convective mixing and its application to micro reactors Proc. 2nd Int. Conf. on Micro- and Minichannels (Rochester NY, USA) pp 781-8

[12] Kockmann N, Engler M and Woias P 2004 Convective mixing and chemical reactions in T-shaped micro reactors Proc. AIChE Annual Meeting p 330

[13] Matsumoto M and Nishimura T 1998 Mersenne twister: a 623-dimensionally equidistributed uniform pseudorandom number generator ACM Trans. Model. Comput. Simul. 8 3-30 